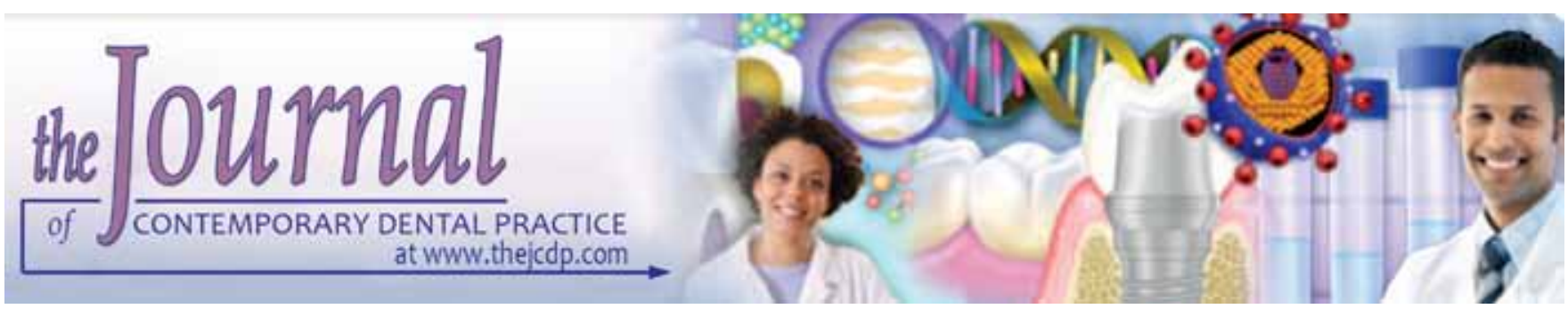

\title{
Periodontal Status in Subjects with Hyperlipidemia and Determination of Association between Hyperlipidemia and Periodontal Health: A Clinicobiochemical Study
}

\author{
TP Shivakumar, Veena Ashok Patil, Manthan H Desai
}

\begin{abstract}
Aim: The aim of this study was to assess the periodontal status in subjects with hyperlipidemia and to determine whether there is any association between hyperlipidemia and periodontitis.

Materials and methods: Sixty subjects with hyperlipidemia and 60 normolipidemic subjects participated in the study. Biochemical parameters assessed included plasma triglyceride, total cholesterol, low density lipoprotein cholesterol (LDL-C) and high density lipoprotein cholesterol (HDL-C) levels. Periodontal parameters assessed included plaque index (PI), probing depth (PD), clinical attachment level (CAL) and percentage of sites with bleeding on probing (BOP).
\end{abstract}

Results: Hyperlipidemic patients showed significantly higher values across the four periodontal parameters. Plasma triglycerides, total cholesterol, LDL-C were significantly and positively associated with $\mathrm{PD}, \mathrm{BOP}$ and $\operatorname{CAL}(p=0.05)$. Whereas HDL-C levels were significantly and negatively associated with PD, BOP and CAL ( $p=0.05)$.

Conclusion: Patients with hyperlipidemia manifested higher values of periodontal parameters compared to control group. Poorly controlled hyperlipidemia had a destructive impact on periodontium. A cyclic relationship exists between serum lipids, periodontitis and systemic health.

Clinical significance: The relationship between periodontium and systemic factors is bidirectional and interlinked. Therefore, dentists and physicians should take this into account while treating patients, aiming for the overall betterment of the patient.

Keywords: Hyperlipidemia, Periodontitis, Lipid profile.

How to cite this article: Shivakumar TP, Patil VA, Desai $\mathrm{MH}$. Periodontal Status in Subjects with Hyperlipidemia and Determination of Association between Hyperlipidemia and Periodontal Health: A Clinicobiochemical Study. J Contemp Dent Pract 2013;14(5):785-789.

Source of support: Nil

Conflict of interest: None declared

\section{INTRODUCTION}

Periodontitis is an inflammatory disease of the supporting tissues of the teeth caused by specific microorganisms or group of specific microorganisms, resulting in progressive destruction of the periodontal ligament and alveolar bone with pocket formation, recession or both. The clinical manifestations of periodontal disease result from a complex interplay between the etiologic agents. Specific bacteria found in the dental plaque and the host tissue factors influence the progression of periodontal disease. Many systemic diseases and disorders have been implicated as risk indicators or risk factors in periodontal disease.

Clinical and basic science research over the past several decades has unveiled a much better understanding of the complexity and pathogenesis of periodontal diseases. It is clear from many studies ${ }^{2}$ that certain systemic conditions may affect the initiation and progression of periodontitis. Systemic disorders affecting neutrophil, monocyte/ macrophage and lymphocyte function result in altered production or activity of host inflammatory mediators. ${ }^{3}$ These alterations may manifest clinically as early onset of periodontal destruction or a more rapid rate of destruction than would occur in the absence of such disorders.

Hyperlipidemia is one such condition with abnormally elevated levels of any or all lipids and/or lipoproteins in the blood and is known to be associated with periodontitis. ${ }^{6}$ Studies ${ }^{4-6}$ have shown that hyperlipidemia causes impairment in receptors, enzymes and response of various cell types. Lipids may interact directly with the macrophage cell membrane, interfering with membrane bound receptors and enzyme systems. Studies have shown that periodontitis which resulted due to hyperlipidemia causes further elevation of serum lipid levels through 'systemic action' of proinflammatory cytokines (TNF- $\alpha / \mathrm{IL}-1 \beta$ ) which leads to further impairment of periodontal tissue response. ${ }^{7-9}$

But, these studies are primarily been conducted in subjects with periodontitis and there are few studies regarding the periodontal conditions of patients with 
hyperlipidemia. The purpose of this study was to evaluate the periodontal status in subjects with hyperlipidemia and to determine whether there is any association between hyperlipidemia and periodontal disease.

\section{MATERIALS AND METHODS}

A cross-sectional case controlled study was designed and conducted at Department of Periodontics, HKE Society's S Nijalingappa Institute of Dental Sciences \& Research, Gulbarga, Karnataka India. The study included 120 subjects. Patients who were diagnosed of having hyperlipidemia from Department of Internal Medicine, Basaveshwara Teaching and General Hospital, Gulbarga, were included in the study. Controls were randomly selected from the patients who came for routine checkup. Sixty subjects who were diagnosed of having hyperlipidemia and 60 normolipidemic subjects were included in the study after biochemical analysis. Verbal and written informed consent was obtained from all subjects prior to their enrollment in the study. Ethical clearance was obtained from the board of ethical committee for the dental college.

The inclusion criteria were as follows:

1. Patients with age $\geq 25$ years.

2. Twenty subjects each with mild, moderate and severe hyperlipidemia.

3. Sixty subjects with normal lipid profile were included in the study.

4. Subjects with $\geq 10$ natural teeth.

The exclusion criteria were as follows:

1. Subjects with the history of systemic disease that affects lipid metabolism, such as:

a. Endocrine disease

- Diabetes mellitus

b. Nephrotic syndrome

- Chronic renal failure

c. Cardiovascular disease

2. Subjects with history of drug treatment for hyperlipidemia.

3. Liver function test up to three times the normal range.
4. Subjects who were pregnant at the time of study.

5. Subjects with history of periodontal treatment 6 months prior to the study.

6. Subjects with history of systemic antibiotic administration within the last 3 months.

The subjects were divided into four groups as follows.

1. Group I: Subjects with mild hyperlipidemia [Total cholesterol: $<200 \mathrm{mg} / \mathrm{dl}$, triglycerides (TRG): 150-199 mg/ dl, low density lipoprotein cholesterol (LDL-C): 100-129 $\mathrm{mg} / \mathrm{dl}$, high density lipoprotein cholesterol (HDL-C): $<40-50 \mathrm{mg} / \mathrm{dl}]$.

2. Group II: Subjects with moderate hyperlipidemia (total cholesterol: 200-240 mg/dl, TRG: 200-499 mg/dl, LDL-C: $130-159 \mathrm{mg} / \mathrm{dl}$, HDL-C: 40-59 mg/dl).

3. Group III: Subjects with severe hyperlipidemia (total cholesterol: >240 mg/dl, TRG: >500 mg/dl, LDL-C: 160-189 mg/dl, HDL-C: $<40 \mathrm{mg} / \mathrm{dl})$.

4. Group $I V$ : Subjects with normal lipid profile (total cholesterol <170-190 mg/dl, TRG: <130-140 mg/dl, LDL-C: 80-90 mg/dl, HDL-C: $<30-40 \mathrm{mg} / \mathrm{dl}$ ).

\section{RESULTS}

The mean standard deviation (SD) values for total cholesterol, TRG and LDL-C were higher in all experimental groups except total cholesterol in mild hyperlipidemic group when compared to controls. The mean value for HDL-C was lower in all experimental groups when compared to controls (Table 1 and Graph 1).

The mean SD values for plaque index (PI), probing depth (PD), bleeding on probing (BOP) and clinical attachment level (CAL) were higher in all three experimental groups when compared to controls (Table 2 and Graph 2).

Total cholesterol, TRG and LDL-C were significantly higher $(\mathrm{p}=0.05)$ in all three experimental groups when compared to controls except for total cholesterol and LDL-C in mild hyperlipidemic groups. The HDL-C level was significantly lower in experimental groups when compared to controls (Table 3 and Graph 3).

\begin{tabular}{lcccc}
\multicolumn{5}{c}{ Table 1: Mean standard deviation of lipid profile of experimental groups and controls } \\
\hline & Control & Mild & Moderate & Severe \\
\hline Total cholesterol & $169.8 \pm 28.76$ & $160.38 \pm 54.4$ & $233.7 \pm 4.75$ & $275.4 \pm 11.25$ \\
Triglyceride & $81.1 \pm 17.82$ & $162.8 \pm 14.21$ & $380.0 \pm 31.09$ & $487.8 \pm 13.99$ \\
HDL cholesterol & $41.6 \pm 1.91$ & $35.8 \pm 3.19$ & $34.6 \pm 2.52$ & $33.4 \pm 2.65$ \\
LDL cholesterol & $101.6 \pm 28.56$ & $118.8 \pm 5.71$ & $153.0 \pm 3.38$ & $178.4 \pm 7.29$ \\
\hline
\end{tabular}

\begin{tabular}{lcccc}
\multicolumn{5}{c}{ Table 2: Mean standard deviation of periodontal parameters for experimental groups and controls } \\
\hline & Control & \multicolumn{1}{c}{ Mild } & Moderate & Severe \\
\hline PI & $1.41 \pm 0.11$ & $1.8 \pm 0.14$ & $1.88 \pm 0.15$ & $2.05 \pm 0.18$ \\
PD & $2.04 \pm 0.08$ & $2.86 \pm 0.19$ & $3.0 \pm 0.24$ & $4.11 \pm 0.32$ \\
BOP & $0.45 \pm 0.18$ & $0.64 \pm 0.08$ & $0.65 \pm 0.07$ & $0.92 \pm 0.08$ \\
CAL & $2.04 \pm 0.08$ & $2.64 \pm 0.16$ & $2.95 \pm 0.20$ & $3.95 \pm 0.31$ \\
\hline
\end{tabular}


Periodontal Status in Subjects with Hyperlipidemia and Determination of Association between Hyperlipidemia

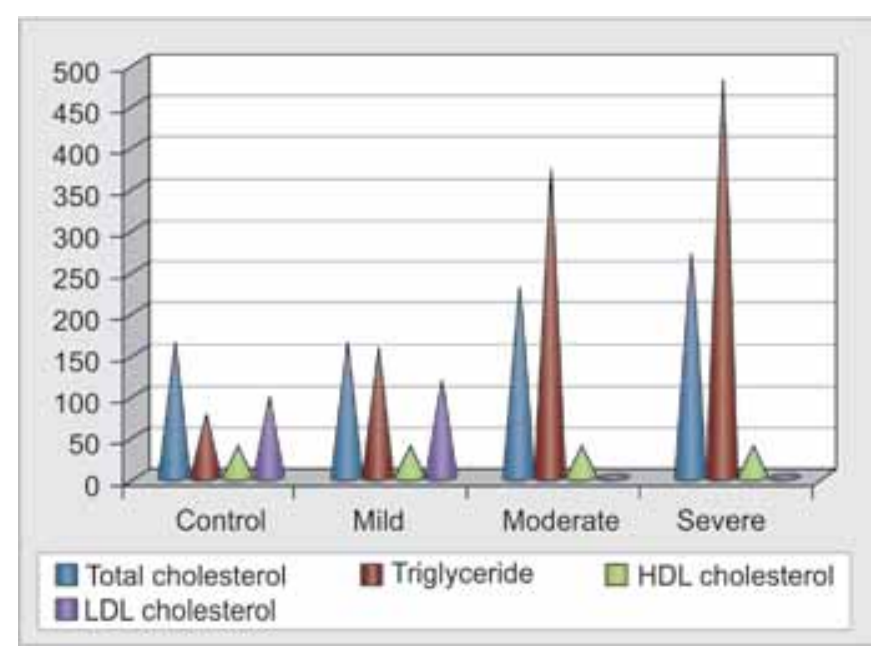

Graph 1: Mean standard deviation of lipid profile of experimental groups and controls

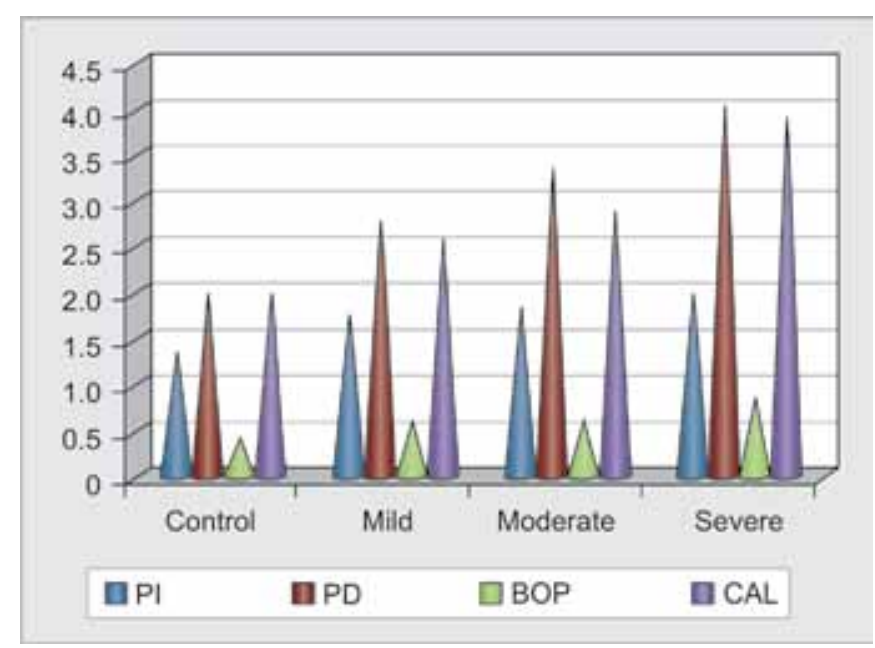

Graph 2: Mean standard deviation of periodontal parameters for experimental groups and controls

PI, PD, BOP and CAL was significantly $(\mathrm{p}=0.05)$ higher in all three experimental groups when compared to controls (Table 4 and Graph 4).

\section{DISCUSSION}

Although the pathogenic role of microbial plaque is unquestionable, several other risk factors that can influence the initiation and progression of periodontitis are still under discussion. The progression and extent of periodontitis are modulated by the bacterial virulence and host immune responses. Certain patients appear to be at a higher risk for periodontal destruction and several risk factors may play an important role in development of periodontitis.

Traditional paradigm has maintained that periodontitis is an oral disease and that the tissue destructive response remains localized within the periodontium, limiting effects of the disease to oral tissues supporting the teeth. ${ }^{10}$ Recent studies ${ }^{11}$ have indicated that periodontitis may produce any number of alterations in the systemic health.

\begin{tabular}{lrcr}
\multicolumn{4}{c}{$\begin{array}{c}\text { Table 3: Student's t-values to compare lipid profile of } \\
\text { experimental groups with the control group }\end{array}$} \\
\hline & Mild & Moderate & Severe \\
\hline Total cholesterol & 0.45 & 6.16 & 10.26 \\
Triglyceride & 10.08 & 16.02 & 23.85 \\
HDL-C & 4.68 & 4.93 & 7.53 \\
LDL-C & 1.77 & 5.36 & 7.82 \\
\hline
\end{tabular}

\begin{tabular}{lrrr}
\multicolumn{4}{c}{ Table 4: Student's t-values to compare the periodontal } \\
parameters of experimental groups with the controls
\end{tabular}

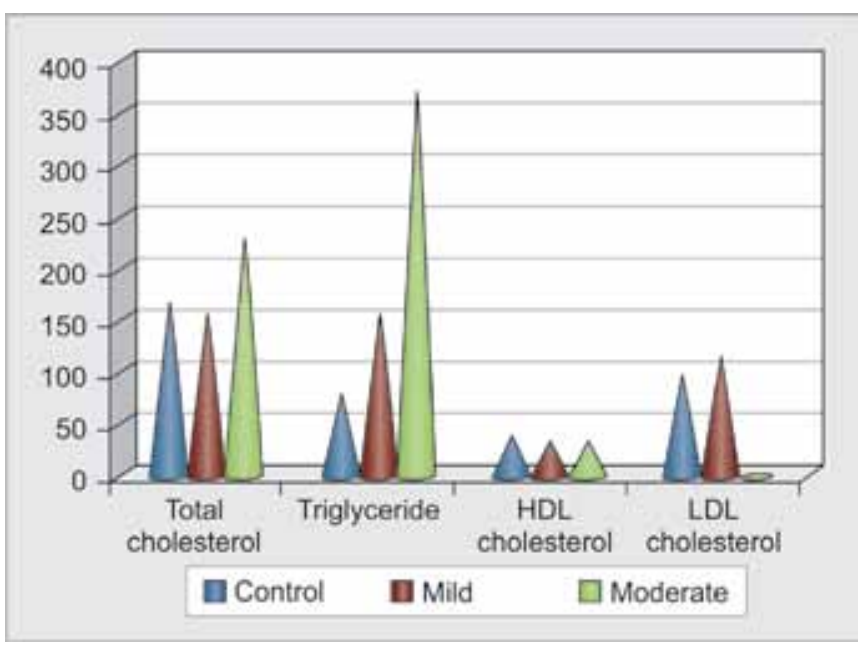

Graph 3: Student's t-values to compare lipid profile of experimental groups with the control group

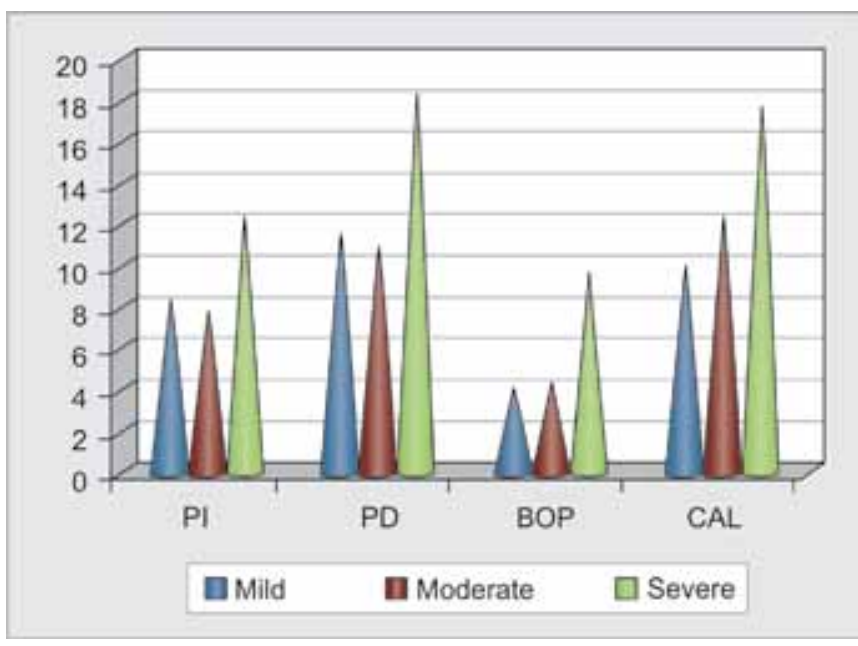

Graph 4: Student's t-values to compare the periodontal parameters of experimental groups with the controls

The results of this study could be explained by the fact that hyperlipidemia which is arising from a high fat diet or other systemic disorders, such as type II diabetes, has 
a dysregulatory effect on the immune cells. Neutrophils exposed to TRG exhibit a significantly increased production of IL-1 $1 /$ superoxide and that PMNs isolated from diabetic and normal patients with high serum LDL/TRG demonstrates impaired chemotaxis, phagocytosis and killing. ${ }^{12,13}$

Hyperlipidemia also causes profound changes in macrophage gene expression. Production of essential polypeptide derived growth factors such PDGF and TGF- $\beta$ were significantly inhibited by exposure to increased level of TRG/LDL. Furthermore, lipids may interact directly with the macrophage cell membrane, interferes with membrane bound receptors. ${ }^{14}$ Monocytes when exposed to high TRG/ LDL level become hyper-responsive. All these changes in the neutrophils, macrophage and monocytes under the influence of increased lipids lead to increased secretion of proinflammatory cytokines, such as IL-1 $\beta /$ TNF- $\alpha .{ }^{15}$

In the case of periodontitis, elevation of these cytokines may be mediated by 'systemic dumping' of locally produced IL- $1 \beta / \mathrm{TNF}-\alpha$ and/or low-level asymptomatic bacteremia/endotoxemia in the absence of systemic complications. ${ }^{16,17}$ It is believed that these cytokines exert effects on lipid metabolism by (a) influencing production of other cytokines, ${ }^{18}$ (b) altering hemodynamics/amino acid utilization of various tissues involved in the lipid metabolism ${ }^{19}$ and (c) modifying the hypothalamic-pituitaryadrenal axis which increases the plasma concentration of cortisol, adrenocorticotropic hormone, adrenaline, noradrenaline, glucagon, etc. ${ }^{20}$ All these factors lead to elevation in serum lipid levels through (a) enhanced hepatic lipogenesis, (b) increased adipose tissue lipolysis/blood flow and (c) increased synthesis and reduced clearance of TRG/ LDL due to reduction in lipoprotein lipase activity.

There are several studies regarding the association between periodontal disease and serum lipid levels, but the results were extremely controversial. Some reports suggested that there is a relationship between cholesterol levels and periodontitis ${ }^{21,22}$ while other reports showed a relationship between TRG levels and periodontitis. ${ }^{23}$

\section{CONCLUSION}

In the present study, we showed convincing evidence that patients with hyperlipidemia had significantly higher values across the four periodontal parameters. Poorly controlled hyperlipidemics showed more gingival inflammation, bleeding on probing and increased pocket depth compared to controls.

In summary, a cyclic relationship exists between serum lipids, tissue response, periodontitis and systemic health, i.e. elevated serum lipids, have dysregulatory effect on the tissue response which in turn affects the extent and progression of periodontitis and in the presence of periodontitis, lipid metabolism will be affected through systemic effect of cytokines.

Additional studies that include more sample size and people with different degrees of hyperlipidemia will provide new contributions to these results. Developing therapeutic approaches should aim at treating both periodontal disease and hyperlipidemia considering the association between the two.

\section{CLINICAL SIGNIFICANCE}

The relationship between periodontium and systemic factors is bidirectional and interlinked. Therefore, dentists and physicians should take this into account while treating patients, aiming for the overall betterment of the patient.

\section{REFERENCES}

1. Newman M, Takei H, Klokkevold P, Carranza F. Carranza's clinical periodontology. 10th ed. Philadelphia: Saunders; 2006. $284 \mathrm{p}$.

2. Offenbacher S. Periodontal diseases: pathogenesis. Ann Periodontol 1996;1:821-878.

3. Page RC. The role of inflammatory mediators in the pathogenesis of periodontal disease. J Periodontol Res 1991;26:230-242.

4. Dutta-Roy AK. Lipid metabolism. Curr Opin Lipidol 1994;5: U9-16.

5. Clark SD, Jump DB. Regulation of gene transcription by polyunsaturated fatty acids. Prog Lipid Res 1993;32:139-149.

6. Stubbs CD, Smith AD. The modification of mammalian membrane polyunsaturated fatty acid composition in relation to membrane fluidity and function. Biochem Biophys Acta 1984;779:89-137.

7. Naock B, Jachmann I, Roscher S, Sieber L, Kopprasch S, Lück C, Hanefeld M, Hoffmann T. Metabolic diseases and their possible link to risk indicators of periodontitis. J Periodontol 2000;71:898-903.

8. Katz J, Chaushu G, Sharabi V. On the association between hypercholesterolemia, cardiovascular disease and severe periodontal disease. J Clin Periodontol 2001;28:865-868.

9. Poh A, Pohl C, Krause S, Gangler P, Loesche W. Hyperlipidemia, atherosclerosis and oral inflammatory diseases. Acta Angiologica 1995;1:133-137.

10. Lacopino AM, Cutler CW. Pathophysiological relationships between periodontitis and systemic disease: recent concepts involving serum lipids. J Periodontol 2000;71:1375-1384.

11. Page RC. The pathobiology of periodontal diseases may affect systemic diseases: inversion of a paradigm. Ann Periodontol 1998;3:108-120.

12. Uhlinger DJ, Cutler CW, Arnold RR, Lambeth JD, Merrill AH. Functional differences in human neutrophils isolated pre and post-prandially. FEBS Lett 1991;286:28-32.

13. Cutler CW, Eke P, Arnold RR, Van Dyke TE. Defective neutrophils function in insulin-dependent diabetes mellitus: a case report. J Periodontol 1991;62:394-401.

14. Chu X, Newman J, Park B, Nares S, Ordonez G, Icopino AM. In vitro alteration of macrophage phenotype and function by serum lipids. Cell Tissue Res 1999;296:331-337.

15. Jovinge S, Ares M, Kallin B, Nilsson J. Human monocytes/ macrophages release TNF- $\alpha$ in response to ox-LDL. Arterioscler Thromb Vasc Biol 1996;16:1573-1579. 
16. Prabhu A, Michalowicz BS, Mathur A. Detection of local and systemic cytokines in adult periodontitis. J Periodontol 1996;67:515-522.

17. Scannapieco FA. Position paper of the American Academy of Periodontology: periodontal disease as a potential risk factor for systemic diseases. J Periodontol 1998;69:841-850.

18. Van der Poll T, Romijn JA, Endert E, Borm JJJ, Buller HR, Sauerwein HP. Tumor necrosis factor mimics the metabolic response to infection in healthy humans. Am J Physiol 1991;261:457-465.

19. Fukushima R, Saito H, Taniwaka K. Different roles of IL-1 and TNF on hemodynamics, amino acid metabolism in dogs. Am J Physiol 1992;262:275-281.

20. Imura H, Fukuta J, Mori T. Cytokines and endocrine function: an interaction between the immune and neuroendocrine systems. Clin Endocrinol 1991;35:107-115.

21. Cutler CW, Shinedling EA, Nunn M, Jotwani R, Kim BO, Nares S, Iacopino AM. Association between periodontitis and hyperlipidemia: Cause or effect? J Periodontol 1999;70: 1429-1434.

22. Morita M, Horiuchi M, Kinoshita Y, Yamamato T, Watanabe T. Relationship between blood triglyceride levels and periodontal status. Community Dent Health 2004;21:32-36.
23. Losche W, Karapetow F, Pohl A, Pohl C, Kocher T. Plasma lipid and blood glucose levels in patients with destructive periodontal disease. J Clin Periodontol 2000;27:537-541.

\section{ABOUT THE AUTHORS}

\section{TP Shivakumar}

Senior Lecturer, Department of Periodontics, Sharavathi Dental College and Hospital, Shimoga, Karnataka, India

\section{Veena Ashok Patil (Corresponding Author)}

Professor and Head, Department of Periodontics, HKE Society's S Nijalingappa Institute of Dental Sciences and Research, Gulbarga Karnataka, India, Phone: +91-9480285089, e-mail: veenaashokpatil@ gmail.com

\section{Manthan H Desai}

Postgraduate Student, Department of Periodontics, HKE Society's S Nijalingappa Institute of Dental Sciences and Research, Gulbarga Karnataka, India 